\title{
Radiotherapy of MRI-detected involved internal mammary lymph nodes in breast cancer
}

Sean Sachdev ${ }^{1 \dagger}$, Chelain R. Goodman ${ }^{1 \dagger}$, Erin Neuschler ${ }^{2}$, Kapila Kalakota ${ }^{3}$, Daniel Cutright ${ }^{1}$, Eric D. Donnelly ${ }^{1}$, John P. Hayes ${ }^{1}$, Adam E. Prescott ${ }^{1}$, Gianna Mirabelli ${ }^{1}$ and Jonathan B. Strauss ${ }^{1 *}$

\begin{abstract}
Background: The internal mammary (IM) lymph node chain, along with the axillary nodal basin, is a first-echelon breast lymphatic draining site. A growing body of evidence supports irradiation of this region in node-positive breast cancer. This study evaluated the effectiveness of radiotherapy in treating magnetic resonance imaging (MRI)-detected abnormal IM lymph nodes in newly-diagnosed non-metastatic breast cancer.

Methods: A structured query was performed on an electronic institutional database to identify women with radiographic evidence of abnormal IM node(s) on breast MRI from 2005 to 2013. Manual review narrowed inclusion to patients with a primary diagnosis of non-metastatic breast cancer with abnormal IM node(s) based on pathologic size criteria and/or abnormal enhancement.

Results: Of the 7070 women who underwent pre-treatment MRI, 19 (0.3\%) were identified on imaging to have a total of 25 abnormal pre-treatment IM lymph nodes, of which 96\% were located in the first two intercostal spaces and $4 \%$ in the third space. A majority of the primary tumors were high-grade (94.7\%) and hormone-receptor negative (73.7\%), while 47. $4 \%$ overexpressed HER-2/neu receptor. Axillary nodal disease was present in $89.5 \%$ of patients, while one patient had supraclavicular involvement. At a median follow-up of 38 months, $31.6 \%$ of patients had developed metastatic disease and $21.1 \%$ had died from their disease. Of the patients who received IM coverage, none had progressive disease within the IM lymph node chain.

Conclusions: Radiologic evidence of pre-treatment abnormal IM chain lymph nodes was associated with advanced stage, high grade, and negative estrogen receptor status. The majority of positive lymph nodes were located within the first two intercostal spaces, while none were below the third. Radiation of the IM chain in combination with modern systemic therapy was effective in achieving locoregional control without surgical resection in this cohort of patients.
\end{abstract}

Keywords: Breast, Breast neoplasms, Lymph nodes, Magnetic resonance imaging, Radiotherapy

\section{Background}

The internal mammary (IM) lymph node chain, along with the axillary nodal basin, is a primary breast lymphatic draining site. [1] IM nodal dissection was routinely pursued in the 1950s due to reports that as many as $33 \%$ of patients had IM nodal involvement on survey biopsies. [2] Multinational trials subsequently revealed that

\footnotetext{
* Correspondence: jonathan.strauss@nm.org

†Equal contributors

${ }^{1}$ Department of Radiation Oncology, Northwestern University Robert H. Lurie Comprehensive Cancer Center, 251 E. Huron Street LC-178, Chicago, IL 60611, USA

Full list of author information is available at the end of the article
}

extended radical (Urban) mastectomy with IM nodal dissection did not improve survival compared to radical (Halstead) mastectomy [3-6], eventually leading to its disuse.

Historically there has been significant controversy regarding when to electively treat the IM nodal chain with radiotherapy. This controversy stems both from uncertainty concerning the therapeutic value of treating the IM chain as well as concern over the incremental additional dose delivered to heart and lung. [7] Recent advances in the delivery of radiotherapy, such as deep inspiration-breath hold, have abrogated the concerns 
about cardiac dose, although the magnitude of the benefit remains unclear. Prospective randomized data have demonstrated that nodal radiotherapy improves locoregional control as well as distant metastasis-free survival and reduces breast cancer mortality. [8, 9] Furthermore, the large absolute overall survival advantage of postmastectomy radiotherapy in node positive women suggests that the inclusion of the regional nodal beds may provide at least part of this observed benefit. [10-13] These reports, however, were based on outcomes of nodal treatment in aggregate; limited data exist regarding the incremental benefit associated with including the IM chain. The Danish Breast Cancer Cooperative Group (DBCG)-IMN study, which prospectively assigned patients with left-sided breast cancer to receive radiation to the IM chain, demonstrated a significant survival benefit of IM chain irradiation. [14] To date, however, there are no randomized clinical trials evaluating this question, and there remains a lack of consensus as to which patients may benefit from the elective radiation of this region. [15-17]

In this study, radiotherapeutic treatment of the IM chain was evaluated in a related setting. Rarely, patients are found to have abnormal pre-treatment IM lymph nodes on imaging. From a large pool of patients who underwent pre-treatment MRI evaluation, patients who were found to have radiologic evidence of internal mammary node involvement were identified. Clinicopathologic data, radiotherapeutic treatment parameters, and clinical outcomes were analyzed to help clarify the effectiveness of radiotherapy and modern systemic therapy in treating involved IM nodes.

\section{Methods}

\section{Patient selection and search query}

Patients were retrospectively identified from an electronic database with records of 7070 women who underwent pre-operative breast MRI from 2005 to 2013 at a single institution. All data collection and analyses were performed after review by and approval of the institutional review board (IRB) and in accordance with medical research principles outlined by the Declaration of Helsinki. [18] Numerous search strings, such as "internal mammary node" and "IM node," were used to identify studies with abnormal internal mammary lymph node(s) as documented by a breast radiologist. A structured query was used to obtain a comprehensive list from which duplicates were excluded.

\section{Imaging, dosimetry, and clinical review}

MR studies were retrieved and manual review narrowed case inclusion to women with a primary diagnosis of non-metastatic breast cancer with abnormal IM node(s) based on size and/or abnormal contrast enhancement as seen on axial fat suppressed T1-weighted and STIR Axial images. Each MRI was individually reviewed with an experienced breast radiologist and a final determination was reached regarding radiographic diagnosis of IM chain involvement. For each patient, the number and size of the involved IM lymph node(s), intercostal space location(s), radiologic evidence of extracapsular extension (ECE), as well as quadrant(s) involved by the primary malignancy were noted. Characteristics of the primary tumor including grade, evidence of lymphovascular invasion (LVI), and hormone receptor profile were recorded. Treatment-related variables including the type of breast surgery and axillary node evaluation as well as the type and sequence of systemic therapy.

All available radiation treatment planning data were de-archived. Dosimetric parameters including prescribed dose, mean dose received, and dose prescribed to $95 \%$ of the treated volume (D95) were recorded for the breast/ chest wall clinical target volume (CTV), axillary and supraclavicular (SCV) nodal chain CTV, and IM nodal chain CTV. The use of separate fields or boosts to cover involved lymph nodes was also documented. Clinical and pathologic stages were assigned per the American Joint Committee on Cancer (AJCC) 7th edition staging criteria. Follow-up date was defined as the last documented note in our institution's electronic medical record system or date of death. Follow-up time was defined as the number of months between date of diagnosis and last follow-up date. Date and location of recurrence, metastasis, or death was documented. Survival curves were plotted as unadjusted Kaplan-Meier estimates.

\section{Results}

Nineteen women with a median age of 52 years (range $=$ 33-88) were identified to have abnormal pre-treatment IM lymph nodes by database screen and subsequent manual review (Table 1). All patients had a clinical tumor classification of T2-4. The pathologic stage ranged from IB to IIIC with the exception of 5 women (26.3\%) who demonstrated complete response after neoadjuvant treatment. The median size of the greatest dimension of the primary tumor as measured clinically was $6.7 \mathrm{~cm}$ (range $=2.10-13.30 \mathrm{~cm}$ ). High histologic grade was assigned to $94.7 \%$ (18/19) of primary tumors, and $52.6 \%(10 / 19)$ demonstrated LVI. ECE of axillary nodal disease was present in $31.6 \%(6 / 19)$ of patients. Fifteen patients $(73.7 \%)$ had hormone-receptor negative tumors, of whom (10/19) were HER-2/neu positive and $7 / 19$ had triple negative disease. Seventeen patients (89.5\%) had identifiable axillary nodal disease, while one patient had both axillary and supraclavicular nodal involvement. 
Table 1 Patient characteristics

\begin{tabular}{|c|c|c|c|c|c|c|c|c|c|c|}
\hline No. & Age & CTN & pTN & No. IM nodes & IM node size & No. Axillary nodes & Inner Quad & LVI & Radiation to IM node & Status \\
\hline 1 & 34 & CT3N3 & ypTON1 & 1 & 1.80 & $1 / 35$ & Yes & Yes & Yes & \\
\hline 2 & 37 & CT4N3 & ypTON1 & 1 & 1.40 & $1 / 24$ & Yes & No & Yes & \\
\hline 3 & 42 & CT3N3 & ypT3N1 & 1 & 2.40 & $1 / 3$ & No & Yes & Unk & Metastatic \\
\hline 4 & 52 & CT3N3 & ypT3N2 & 2 & $1.40,0.90$ & $8 / 8$ & No & Yes & Yes & \\
\hline 5 & 57 & CT3N3 & ypTON3 & 1 & 1.40 & $23 / 28$ & Yes & Yes & Yes & Metastatic \\
\hline 6 & 61 & CT2N2 & ypT1No & 2 & $1.40,1.30$ & $1 / 1$ & No & Unk & Unk & \\
\hline 7 & 62 & $\mathrm{CT} 2 \mathrm{~N} 3$ & ypT1NO & 1 & 1.00 & $0 / 13$ & No & Yes & Yes & \\
\hline 8 & 65 & CT3N2 & ypT3N1 & 2 & $1.70,2.90$ & $2 / 24$ & Yes & Yes & No & LRR to IM chain \\
\hline 9 & 66 & cT2N3 & ypT1NO & 1 & 2.60 & $0 / 3$ & No & No & Yes & \\
\hline 10 & 34 & cT2N3 & ypTONO & 1 & 2.10 & $0 / 18$ & Yes & No & Yes & \\
\hline 11 & 44 & CT3N2 & ypTONO & 2 & $2.00,0.60$ & $0 / 1$ & Yes & Unk & Yes & \\
\hline 12 & 49 & $\mathrm{CT} 2 \mathrm{~N} 2$ & ypTONO & 2 & $1.00,1.00$ & $0 / 20$ & No & No & Partial & \\
\hline 13 & 50 & CT3N2 & ypTONO & 1 & 0.90 & $0 / 5$ & Yes & No & Yes & Metastatic \\
\hline 14 & 52 & CT3N2 & ypTONO & 1 & 0.60 & $0 / 15$ & No & Unk & Yes & \\
\hline 15 & 43 & $\mathrm{CT} 2 \mathrm{~N} 2$ & pT2N1 & 1 & 3.00 & $2 / 5$ & No & Yes & Yes & LRR to tumor bed \\
\hline 16 & 49 & $\mathrm{CT} 2 \mathrm{~N} 2$ & $\mathrm{pT} 2 \mathrm{~N} 2$ & 1 & 0.50 & $4 / 16$ & No & No & Unk & \\
\hline 17 & 52 & CT3N3 & $\mathrm{pT} 2 \mathrm{~N} 2$ & 1 & 0.40 & $8 / 22$ & No & Yes & Yes & \\
\hline 18 & 53 & CT3N2 & ypTxN2 & 2 & $0.70,0.70$ & $5 / 8$ & Yes & Yes & Unk & Metastatic \\
\hline 19 & 88 & CT3N3 & pT3N3 & 1 & 0.40 & $18 / 35$ & No & Yes & Yes & Metastatic \\
\hline
\end{tabular}

No Number, cTN Clinical Tumor and Nodal classification, pTN Pathologic tumor and nodal classification, yp Pathologic tumor and nodal classification following neoadjuvant chemotherapy, Quad Quadrant, LVI Lymphovascular invasion, LRR Locoregional recurrence; Unk Unknown

Twenty-five abnormal lymph nodes were radiographically identified in total. Twelve (48\%), twelve (48\%), and two $(4 \%)$ nodes were located in the first three intercostal spaces, respectively (Fig. 1). No involvement was found below the 3rd intercostal space. All IM nodal involvement was ipsilateral to the known breast cancer. Eleven women (57.9\%) had malignancies that did not cross into the inner quadrant of the involved breast. Twenty-three of 25 nodes identified (92.0\%) were $\geq 0.5 \mathrm{~cm}$ in at least one dimension. The mean short and long axis lengths were 0.82 (standard deviation $(\mathrm{STD})=0.48 \mathrm{~cm}$ ) and $1.36 \mathrm{~cm}(\mathrm{STD}=0.77 \mathrm{~cm})$, respectively. The mean short axis to long axis ratio $(\mathrm{S} / \mathrm{L})$ was $0.63(\mathrm{STD}=0.19)$. The mean volume was $1.184 \mathrm{~cm}^{3}$ (range $=0.02-5.53 \mathrm{~cm}^{3}$ ) as calculated by the ellipsoid formula.

All but one patient (94.7\%) received chemotherapy; $78.9 \%(15 / 19)$ of patients received neoadjuvant chemotherapy and $15.8 \%(3 / 19)$ were treated with postoperative chemotherapy (Table 1). Of those patients treated with neoadjuvant chemotherapy, 67\% (10/15) demonstrated a pathologic complete response. All but one patient with an estrogen receptor (ER)-positive tumor (3/4) received adjuvant anti-endocrine therapy, while all Her2-overexpressing patients received adjuvant trastuzumab.

Eighteen patients received adjuvant radiation to the breast or chest wall with photons (6-10 Mega-volts

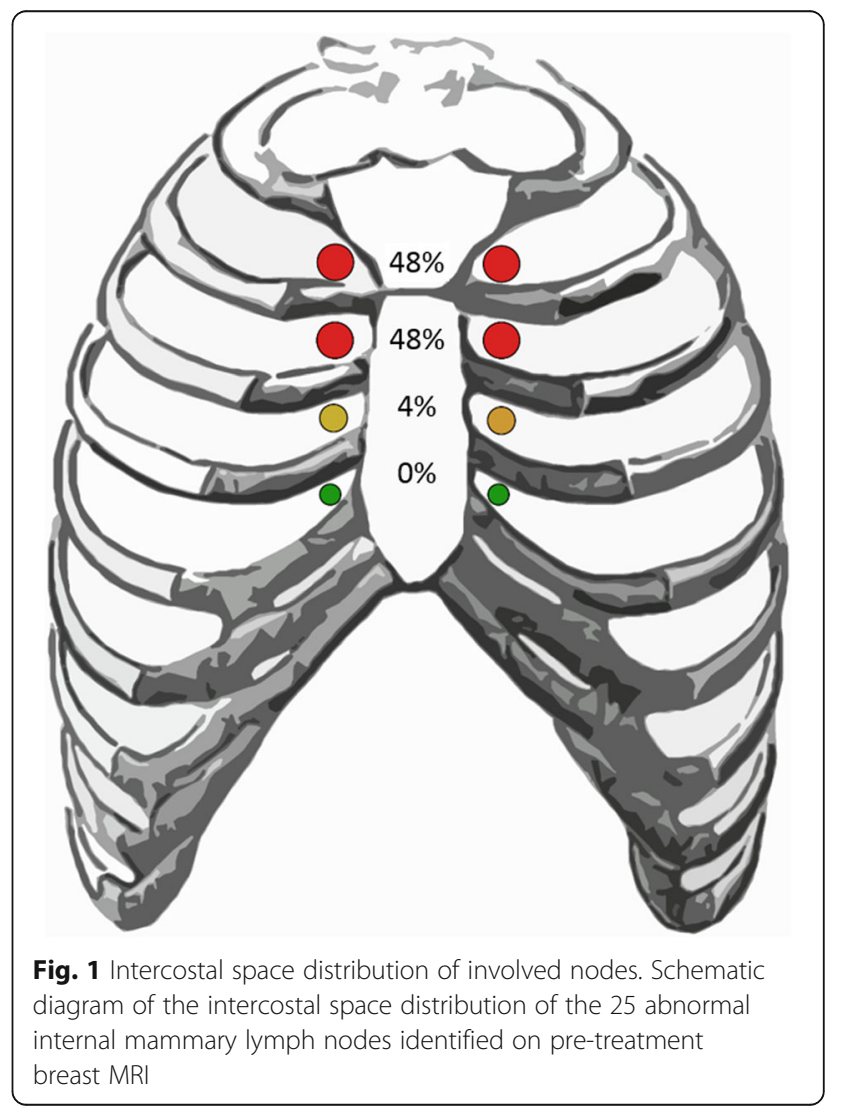


(MV)) using tangent beams with or without matched electron fields (9-12 Megaelectron-volts $(\mathrm{MeV})$ ); one patient was treated with intensity modulated radiotherapy technique. Seventeen patients $(89.4 \%)$ were treated to the axillary and supraclavicular lymph node basins with photons using oblique fields (6-18 MV). Boosts to the mastectomy scar or IM nodal chain were given using electrons (9-20 MeV). Of the 15 patients for whom dose prescriptions were available, the median dose to the breast or chest wall was 50.4 Gray (Gy;range $=45$ $50.4 \mathrm{~Gy}$ ) prior to a median 10Gy boost (range $=0-16 \mathrm{~Gy}$ ) to the surgical bed or mastectomy scar. The median dose to the axillary/supraclavicular basin was 50.4Gy (range $=45-50.4$ Gy). $73.6 \%$ of patients $(14 / 19)$ received dedicated treatment of the IM nodal chain to a median dose of 50.4Gy (range $=45-50.4 \mathrm{~Gy}$ ), while coverage of the IM chain was unknown for 4 patients. A single patient was treated at an outside institution without coverage of the IM chain. Four of the 14 patients who were treated to the IM nodal chain (28.6\%) were prescribed a boost to a median dose of 14.0Gy (range $=10-14 \mathrm{~Gy}$ ). The median total dose prescribed to the IM nodal chain was 50.4Gy (range $=45-64.4 \mathrm{~Gy}$ ). The median mean dose received to the IM nodal chain was 53.5Gy (range = 31.1-75.8Gy). The median D95 was 45.8Gy (range $=$ 12.3-70.8Gy). A representative MRI and treatment plan of a dedicated 14Gy boost to an involved IM node is shown in Fig. 2.

After a median follow-up of 38 months (Interquartile range $=30.0-53.3), 18$ of $19(94.7 \%)$ patients maintained local control of the IM nodal chain and 17 of 19 (89.4\%) were free from locoregional recurrence. No patient that received dedicated treatment of the IM chain experienced a recurrence or progression at the site of the involved node(s). One patient who did not receive coverage of the IM chain developed progressive disease within the IM chain as well as at distant sites, and died 28 months following diagnosis. One patient experienced a local recurrence at the lumpectomy site 28 months following her original diagnosis. Of all 19 patients, 6 (31.6\%) developed metastatic progression to the brain, lung, and/or bone, while 4 (21.1\%) died from their disease by the time of last follow-up (Table 2). Of the 14 patients who received dedicated radiation to the IM chain, 13 (96.9\%) achieved locoregional control, while 3 (21.4\%) developed metastatic disease, and 2 (14.3\%) died (Table 2). Since median survival was not observed, restricted mean survival times were estimated using the Kaplan-Meier method. Restricted mean overall survival was 76.7 months $(95 \%$ Confidence Interval $(\mathrm{CI})=61.0$ 92.4), restricted mean locoregional recurrence free survival was 86.0 months $(95 \% \mathrm{CI}=74.2-97.8)$, and restricted mean disease free survival was 65.2 months $(95 \% \mathrm{CI}=47.8-82.6)$.

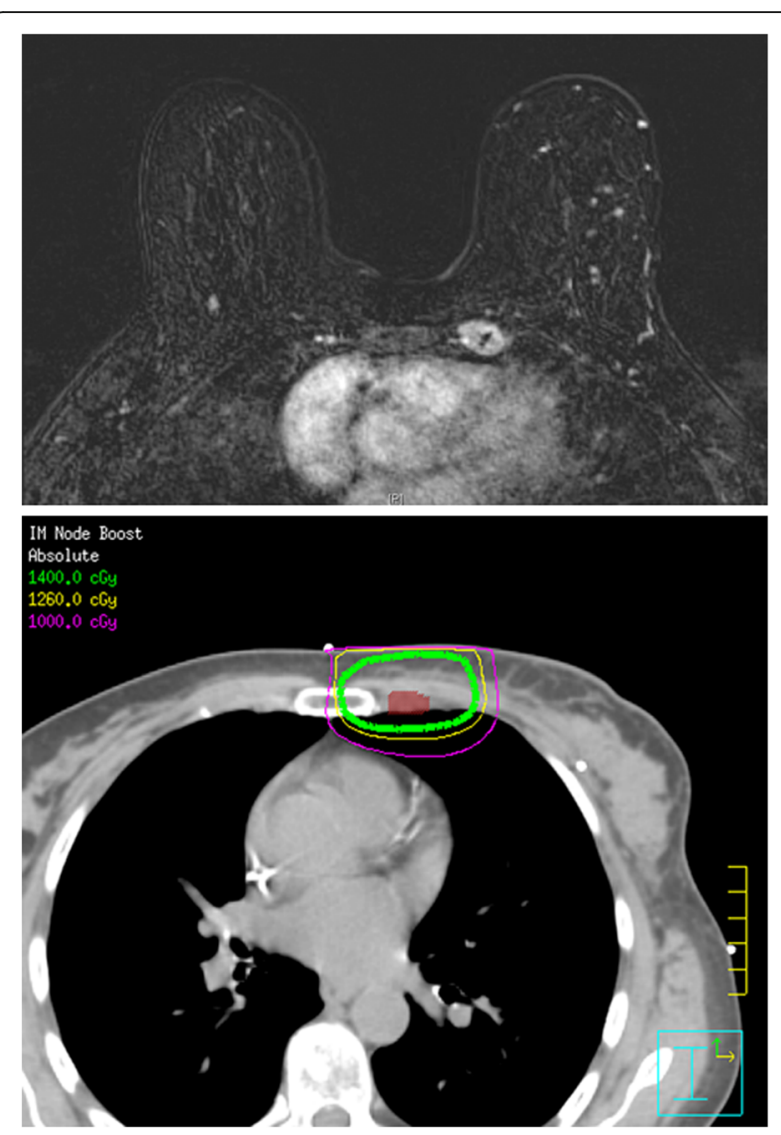

Fig. 2 Representative MRI and treatment plan. Representative internal mammary node identified on MRI (Top) and treatment plan of dedicated 14Gy boost (Bottom). Maroon contour = IM node GTV

\section{Discussion}

The inclusion of the IM nodal chain as part of regional nodal irradiation for patients with breast cancer has been historically controversial in part due to the difficulty in detecting IM nodal involvement. As breast MRIs are increasingly being performed prior to treatment, radiologic identification of involved IM lymph nodes is providing a new window into the phenomenon of IM nodal involvement. [19] In this single institution analysis of 7070 women who underwent pre-treatment MRI, 19 patients were identified by search query and manual review as having clinical involvement of the IM lymph node chain. The cases identified tended to skew towards a more clinically advanced and/or aggressive tumor phenotype. All patients had clinical T2-T4 primary tumors and all but 2 had known axillary or supraclavicular disease. These cases also skewed toward more aggressive subtypes as $94.7 \%$ were grade $3,73.7 \%$ were hormone receptor-negative, and $52.6 \%$ demonstrated overexpression of the Her-2/Neu receptor. The vast majority (96\%) of the involved IM nodes were located in the first 2 intercostal spaces. All but one patient received chemotherapy, and all Her-2/Neu positive patients received 
Table 2 Patient outcomes

\begin{tabular}{lllll}
\hline Event & All patients $(N=19)$ & IM radiation $(N=14)$ & No IM radiation $(N=1)$ & Unknown IM radiation $(N=4)$ \\
\hline IM chain recurrence & $1(5.3 \%)^{\mathrm{a}}$ & $0(0 \%)$ & $1(100 \%)$ & $0(0 \%)$ \\
Locoregional recurrence & $2(10.5 \%)$ & $1(7.1 \%)$ & $1(100 \%)$ & $0(0 \%)$ \\
Distant recurrence & $6(31.6 \%)$ & $3(21.4 \%)$ & $1(100 \%)$ & $2(50.0 \%)$ \\
Death & $4(21.1 \%)$ & $2(14.3 \%)$ & $1(100 \%)$ & $1(25.0 \%)$ \\
\hline
\end{tabular}

IM Internal mammary. ${ }^{\text {a }}$ Did not receive radiation to IM chain

Herceptin. Of the patients who received dedicated radiation to the IM chain, none experienced a recurrence or progression at the site of clinical nodal disease. The sole patient with progression of IM nodal disease had not received radiotherapy to the IM nodal chain. One patient experienced recurrence at the lumpectomy bed. Systemic control, however, remained problematic, as six patients (31.6\%) developed distant disease and four (21.1\%) died within 1-3 years of diagnosis.

In a large surgical (pathologic) series of women who had undergone an extended mastectomy, involvement of the IM region portended significantly worse outcomes: disease-free-survival was $23 \%$ at 20 years compared to $76 \%$ in IM-negative patients. [20] Our results corroborate the finding that IM nodal involvement portends a worse prognosis, although improved staging and systemic therapy, among other advances, account for far superior outcomes in this series as compared to historical data. There are relatively few reports of IM nodal involvement identified on imaging, however, our data appear to be congruous with those of other institutions. Lee et al. demonstrated that malignant IM lymph nodes identified on MRI had a short/long (S/L) axis ratio that was significantly greater than the ratio for benign IM nodes $(0.45 \pm 0.10$ vs $0.59 \pm 0.17)$. [21] The mean S/L ratio of the 26 nodes identified in our series $(0.63 \pm 0.19)$ is consistent with this report. Bellon et al. reported on outcomes of seven women with locally advanced breast cancer and increased uptake in the IM nodes seen on positron emission tomography. [22] Follow-up data were available on 6 of these seven women; all three who received no IM nodal radiotherapy experienced IM nodal failure. In conjunction with our series, this point highlights the need to include involved IM nodes in the radiotherapy field. There was an association between large tumor size and IM nodal involvement, a finding that mirrors the more advanced tumor classification seen in our series. Zhang et al. reported on 112 women with involved IM nodes as seen on a variety of imaging studies. [23] In that series, the vast majority of patients (87\%) had T2-T4 tumors, almost all (91\%) had involved axillary and/or SCV nodes, $75 \%$ were grade 3, and 56\% were ER-negative. Five-year IM nodal control in this group, most of whom received IM nodal radiotherapy, was $84 \%$. These patient and tumor variables are similar to those seen in our study. The high rate of IM nodal control highlights the effectiveness of the combination of targeted radiotherapy and modern systemic therapy.

The results of the present study, in combination with other reports, have interesting implications. First, they validate the current understanding that the upper 3 intercostal spaces of the ipsilateral IM nodal chain account for the vast majority of involved IM nodes and most of those lie in the upper 2 spaces. Thus, if elective IM nodal radiotherapy is to be used, then inclusion of the upper 3 intercostal spaces may be sufficient. Intriguingly, our series identified a preponderance of ER-negative tumors in the patients presenting with IM nodal involvement. This mirrors a planned subset analysis of the MA-20 trial, which found a sizable survival advantage for the addition of nodal radiotherapy that was limited to ER-negative patients. [8] Although patients within the MA-20 trial did not have clinically involved IM nodes, MRI staging was not performed and nodes may have been clinically occult but radiologically suspicious. If validated in other studies, this finding could support the use of elective nodal radiotherapy in these patients. As in our series, Zhang et al. found a high rate of regional control with radiotherapy. This is attributable to the remarkable synergy of accurate modern radiotherapy with 3-dimensional (3D) planning in combination with increasingly effective and targeted systemic therapy. Given the efficacy of these treatment modalities and the high morbidity associated with surgical excision of the IM nodal chain, definitive irradiation of involved IM nodes, when identified, may therefore be the optimal choice of treatment compared to surgery. [24]

There are several limitations to the present study. As a retrospective review, there is an inherent selection bias regarding case identification and are limited by the paucity of identifiable patients from a single institution. Our sample size was further limited as very few women receive MR imaging at our institution as part of the workup for a breast malignancy; indeed, some of the patients included in our cohort received an MR for screening purposes. The overall rate of IM nodal involvement identified in this series is therefore not generalizable to a population with known breast cancer. 


\section{Conclusions}

In summary, in this series a majority of patients with involved IM nodes as identified on pre-treatment MRI had advanced primary tumors that were of aggressive grade and tumor subtype. Involved nodes were limited to the upper three intercostal spaces with the majority found within the first two spaces. IM nodal chain control was achieved for all patients for whom the IM chain was irradiated. A relatively high risk of distant metastatic spread, however, persisted in this population. The combination of modern systemic therapy with radiation including the IM nodes may therefore be sufficient for achieving locoregional control in patients with either clinical evidence of or occult involvement of the IM chain.

\section{Abbreviations}

3D: 3-dimensional; AJCC: American Joint Committee on Cancer; Cl: Confidence Interval; CTV: Clinical target volume; DBCG: Danish Breast Cancer Cooperative Group; ECE: Extracapsular extension; ER: Estrogen receptor; Gy: Gray; IM: Internal Mammary; IRB: Institutional Review Board; LVI: Iymphovascular invasion; MeV: Megaelectron-volt; MRI: Magnetic Resonance Imaging; MV: Mega-volt; SCV: Supraclavicular

\section{Acknowledgments}

A preliminary presentation of these data won the 2015 Lasky Foundation Breast Cancer Research Award competition at Chicago Radiologic Society. Further data were presented at the 57th Annual Meeting of the American Society for Radiation Oncology (ASTRO), October 14-17, 2015, San Francisco, CA.

\section{Funding}

None.

\section{Availability of data and materials}

The datasets generated during and/or analyzed during the current study are not publicly available due to patient confidentiality but are available from the corresponding author on reasonable request.

\section{Authors' contributions}

AP and GM submitted the IRB application and worked with the Northwestern Memorial Hospital Enterprise Data Warehouse (EDW) to identify eligible patients. SS, KK, ED, and $\mathrm{JH}$ were involved in study design and contributed significantly to the editing of the manuscript. EN individually reviewed each MRI. DC obtained and analyzed the dosimetric data. CG, SS, JBS analyzed and interpreted the patient data and wrote the manuscript. All authors read and approved the final manuscript.

\section{Ethics approval and consent to participate}

All data collection and analyses were performed after review by and approval of the institutional review board (IRB) and in accordance with medical research principles outlined by the Declaration of Helsinki.

\section{Consent for publication}

Not applicable.

\section{Competing interests}

The authors declare that they have no competing interests.

\section{Publisher's Note}

Springer Nature remains neutral with regard to jurisdictional claims in published maps and institutional affiliations.

\section{Author details}

'Department of Radiation Oncology, Northwestern University Robert H. Lurie Comprehensive Cancer Center, 251 E. Huron Street LC-178, Chicago, IL 60611, USA. ${ }^{2}$ Division of Breast Imaging, Department of Radiology,
Northwestern University Feinberg School of Medicine, 676 N. St Clair Street \#800, Chicago, IL 60611, USA. ${ }^{3}$ Radiation Oncology Consultants Ltd., 120 N. Oak Street, Hinsdale, IL 60521, USA.

Received: 28 August 2017 Accepted: 30 November 2017

Published online: 19 December 2017

\section{References}

1. Chen RC, Lin NU, Golshan M, Harris JR, Bellon JR. Internal mammary nodes in breast cancer: diagnosis and implications for patient management - a systematic review. J Clin Oncol. 2008;26:4981-9.

2. Handley RS, Thackray AC. Invasion of internal mammary lymph nodes in carcinoma of the breast. Br Med J. 1954;1:61-3.

3. Lacour J, Le M, Caceres E, Koszarowski T, Veronesi U, Hill C. Radical mastectomy versus radical mastectomy plus internal mammary dissection. Ten year results of an international cooperative trial in breast cancer. Cancer. 1983;51:1941-3.

4. Meier P, Ferguson DJ, Karrison T. A controlled trial of extended radical versus radical mastectomy. Ten-year results. Cancer. 1989;63:188-95.

5. Morimoto T, Monden $\mathrm{Y}$, Takashima S, Itoh S, Kimura T, Yamamoto H, Kitamura M, Inui K, Tanaka N, Nagano T, et al. Five-year results of a randomized clinical trial comparing modified radical mastectomy and extended radical mastectomy for stage $\|$ breast cancer. Surg Today. 1994:24:210-4.

6. Veronesi U, Valagussa P. Inefficacy of internal mammary nodes dissection in breast cancer surgery. Cancer. 1981:47:170-5.

7. Macrie BD, Donnelly ED, Hayes JP, Gopalakrishnan M, Philip RT, Reczek J, Prescott A, Strauss JB. A cost-effective technique for cardiac sparing with deep inspiration-breath hold (DIBH). Phys Med. 2015;31:733-7.

8. Whelan TJ, Olivotto IA, Parulekar WR, Ackerman I, Chua BH, Nabid A, Vallis KA, White JR, Rousseau P, Fortin A, et al. Regional nodal irradiation in early-stage breast cancer. N Engl J Med. 2015;373:307-16.

9. Poortmans PM, Collette S, Kirkove C, Van Limbergen E, Budach V, Struikmans H, Collette L, Fourquet A, Maingon P, Valli M, et al. Internal mammary and medial Supraclavicular irradiation in breast cancer. N Engl J Med. 2015;373:317-27

10. Overgaard M, Jensen MB, Overgaard J, Hansen PS, Rose C, Andersson M, Kamby C, Kjaer M, Gadeberg CC, Rasmussen BB, et al. Postoperative radiotherapy in high-risk postmenopausal breast-cancer patients given adjuvant tamoxifen: Danish breast cancer cooperative group DBCG 82C randomised trial. Lancet. 1999;353:1641-8.

11. Overgaard M, Hansen PS, Overgaard J, Rose C, Andersson M, Bach F, Kjaer M, Gadeberg CC, Mouridsen HT, Jensen MB, Zedeler K. Postoperative radiotherapy in high-risk premenopausal women with breast cancer who receive adjuvant chemotherapy. Danish breast cancer cooperative group 82b trial. N Engl J Med. 1997:337:949-55.

12. Ragaz J, Olivotto IA, Spinelli JJ, Phillips N, Jackson SM, Wilson KS, Knowling MA, Coppin CM, Weir L, Gelmon K, et al. Locoregional radiation therapy in patients with high-risk breast cancer receiving adjuvant chemotherapy: 20year results of the British Columbia randomized trial. J Natl Cancer Inst. 2005;97:116-26

13. McGale P, Taylor C, Correa C, Cutter D, Duane F, Ewertz M, Gray R, Mannu G, Peto $\mathrm{R}$, Whelan $\mathrm{T}$, et al. Effect of radiotherapy after mastectomy and axillary surgery on 10-year recurrence and 20-year breast cancer mortality: metaanalysis of individual patient data for 8135 women in 22 randomised trials. Lancet. 2014;383:2127-35.

14. Thorsen LB, Offersen BV, Dano H, Berg M, Jensen I, Pedersen AN, Zimmermann SJ, Brodersen HJ, Overgaard M, Overgaard J. DBCG-IMN: a population-based cohort study on the effect of internal mammary node irradiation in early node-positive breast cancer. J Clin Oncol. 2016;34:314-20.

15. Hennequin C, Bossard N, Servagi-Vernat S, Maingon P, Dubois JB, Datchary J, Carrie C, Roullet B, Suchaud JP, Teissier E, et al. Ten-year survival results of a randomized trial of irradiation of internal mammary nodes after mastectomy. Int J Radiat Oncol Biol Phys. 2013;86:860-6.

16. Chang JS, Park W, Kim YB, Lee IJ, Keum KC, Lee CG, Choi DH, Suh CO, Huh SJ. Long-term survival outcomes following internal mammary node irradiation in stage II-III breast cancer: results of a large retrospective study with 12-year follow-up. Int J Radiat Oncol Biol Phys. 2013;86:867-72.

17. Jagsi $R$, Pierce L. Radiation therapy to the internal mammary nodal region in breast cancer: the debate continues. Int J Radiat Oncol Biol Phys. 2013;86:813-5. 
18. World Medical Association declaration of Helsinki. Recommendations guiding physicians in biomedical research involving human subjects. JAMA. 1997;277:925-6.

19. Esserman L, Hylton N, Yassa L, Barclay J, Frankel S, Sickles E. Utility of magnetic resonance imaging in the management of breast cancer: evidence for improved preoperative staging. J Clin Oncol. 1999:17:110-9.

20. Sugg SL, Ferguson DJ, Posner MC, Heimann R. Should internal mammary nodes be sampled in the sentinel lymph node era? Ann Surg Oncol. 2000;7:188-92.

21. Lee HW, Kim SH. Breast magnetic resonance imaging for assessment of internal mammary lymph node status in breast cancer. J Breast Cancer. 2016;19:191-8.

22. Bellon JR, Livingston RB, Eubank WB, Gralow JR, Ellis GK, Dunnwald LK Mankoff DA. Evaluation of the internal mammary lymph nodes by FDG-PET in locally advanced breast cancer (LABC). Am J Clin Oncol. 2004;27:407-10.

23. Zhang YJ, Oh JL, Whitman GJ, lyengar P, Yu TK, Tereffe W, Woodward WA, Perkins G, Buchholz TA, Strom EA. Clinically apparent internal mammary nodal metastasis in patients with advanced breast cancer: incidence and local control. Int J Radiat Oncol Biol Phys. 2010;77:1113-9.

24. Sacchini G, Borgen PI, Galimberti V, Veronesi P, Zurrida S, Luini A, Spaggiari L, Cody HS 3rd, Veronesi U. Surgical approach to internal mammary lymph node biopsy. J Am Coll Surg. 2001;193:709-13.

\section{Submit your next manuscript to BioMed Central} and we will help you at every step:

- We accept pre-submission inquiries

- Our selector tool helps you to find the most relevant journal

- We provide round the clock customer support

- Convenient online submission

- Thorough peer review

- Inclusion in PubMed and all major indexing services

- Maximum visibility for your research

Submit your manuscript at www.biomedcentral.com/submit 